\title{
Optimization of the Shape of Axi-Symmetric Rubber Bumpers
}

\author{
Tamás Mankovits $1{ }^{1}{ }^{*}$ - Tamás Szabó 2 - Imre Kocsis ${ }^{1}$ - István Páczelt ${ }^{2}$ \\ 1 University of Debrecen, Faculty of Engineering, Hungary \\ 2 University of Miskolc, Faculty of Mechanical Engineering, Hungary
}

The rubber bumpers built into the air-spring structures of buses perform a number of critical tasks. Consequently, designing their shape requires considerable effort. This paper presents a novel solution for determining the required characteristics of axi-symmetric rubber parts, which can efficiently be used in practice.

The procedure is based on the finite element method (FEM) and the support vector regression (SVR) model. A finite element code developed by the authors and based on a three-field functional is used for the rapid and appropriately accurate calculation of the characteristics of rubber bumpers. A rubber shape is evaluated via the work difference and the area between the desired and the actual load-displacement curves. The objective of shape optimization is to find the geometry where the work difference is under a specified limit. The tool of optimization is the SVR method, which provides the regression function for the work difference. The minimization process of the work-difference function leads to the optimum design parameters. The efficiency of the method is verified by numerical examples.

Keywords: shape optimization, rubber bumper, support vector regression, finite element method

\section{INTRODUCTION}

Designing the suspension systems of vehicles is a demanding engineering task. Currently, driving stability is ensured by electronically controlled active suspension systems. The objectives set in the course of designing include improving travel comfort, decreasing the dynamic loading of the wheels and decreasing the suspension workspace [1]. Similar possibilities are offered by the use of air springs. The rubber bumpers (Fig. 1) built into the air springs of buses perform several crucial functions, such as working together with the air spring as a secondary spring, thus modifying the original characteristics of the air spring when pressed together (characteristics of the dotted and dashed lines in Fig. 2). When the bus is in a stationary position and settles to the ground, the static weight of the chassis and the body rests on the bumper; in this case, the solid line characteristic is active. If the fibre-reinforced bellows of the air spring wears through while the bus is running, the vehicle can safely reach the nearest garage at a limited speed while bouncing on the bumper; no additional damage will occur. It prevents metal-on-metal collision at large dynamic impulses and absorbs the impulse. These rubber bumpers are subject to compressive stress, for which the characteristics show a progressive feature.

It is a fundamental requirement that they should have a specified load-displacement curve under load; setting this objective results in a shape optimization task. The aim of the optimization is to achieve a specified characteristic by means of the geometric design of the rubber bumper while the material characteristics remain the same.

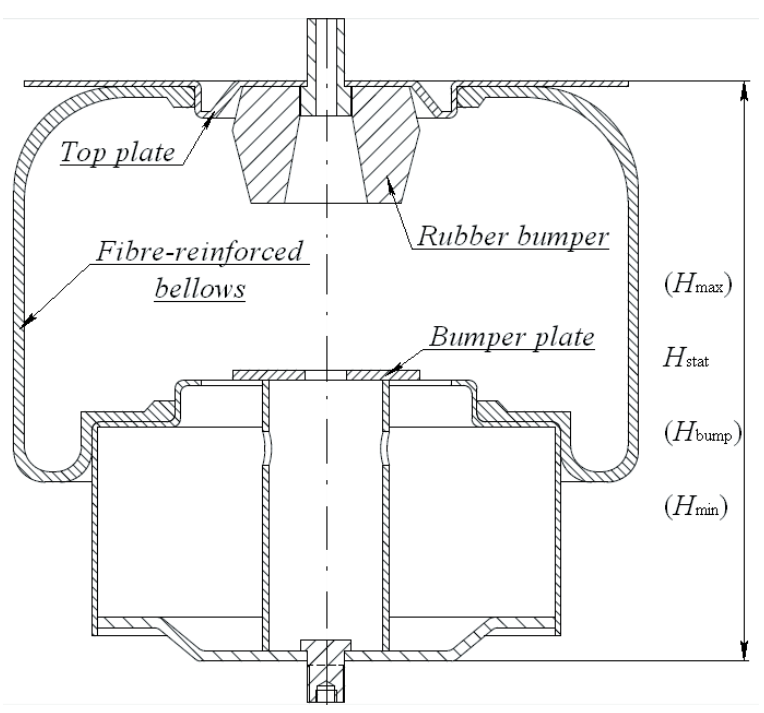

Fig. 1. The air spring

The literature does not devote much room to the examination of the rubber bumpers of air springs. Another area for which rubber mounts are used is the flexible support of engines, on which several works have been published. Estimation of the fatigue life of rubber springs, rubber mounts and air springs is carried out in [2] to [5], respectively. In [6], extensive studies on the rubber mounts of engines are performed using the finite element method. Several authors have formulated shape optimization for the specified stiffness of rubber mounts, in which the analysis was done using commercial finite element software or a finite element code of their own development. The stiffness of rubber mounts in three directions on the basis of parameter examinations is optimized in [7]. Optimization based on sensitivity analysis using a 
special purpose finite element code is performed in [8] for material properties and shape, where stiffness was also taken into consideration. Determining the shape was done with the aim of minimizing the volume of the rubber part. Rubber mounts undergoing shear are modelled with the finite element method in [9], and the effects of the geometric design and the load on the stiffness of the part are also investigated. For the purpose of minimizing the cross-sectional area and the maximum stress of the rubber mount and for that of maximizing the life cycle, shape optimization using an Ogden-type material model and commercial finite element software is applied in [10]. Several objective functions in a system where the optimization had several stages are handled. A back-propagation neural network (BPN) is used to find the connection between the input and output data and then a micro-genetic algorithm (MGA) is used for global optimization. A large number of finite element running results are used as learning points. The experience gained from the above works also easily lends itself to examining the rubber bumper of air springs.

Our research intends to determine the behaviour of rubber bumpers in the complete range of operation, and thus the aim of the shape optimization is to achieve the specified spring characteristics. Since there is no active control in the rubber bumpers, shape optimization may provide the required loaddisplacement curve. In connection with the objective set, achieving the aim of the optimization will require an efficient load-displacement calculation, which is performed using the finite element method. This is the purpose served by the finite element program prepared for the examination of axi-symmetric rubber parts, which can be conveniently fit to the shape optimization procedure.

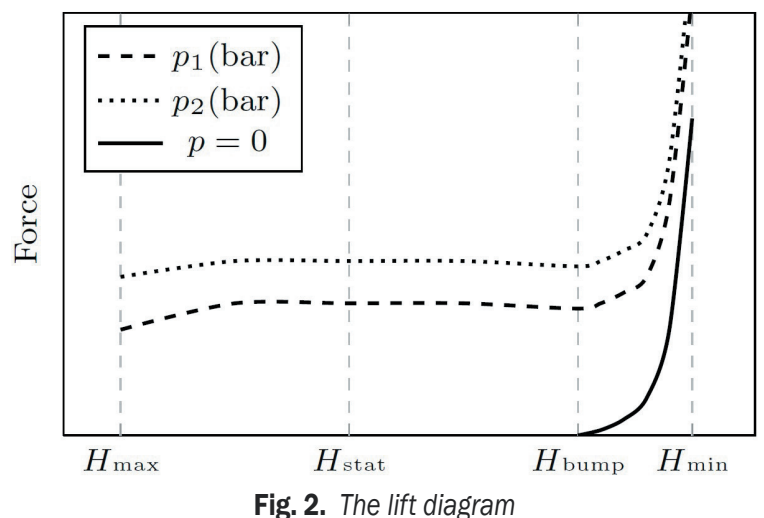

The support vector regression (SVR) proposed by [11] is a widely used application of support vector machines (SVM) for regression problems, e.g. in optimization models. A great number of applications can be found in the fields of materials science, chemistry, economics and data procession, where connections are sought between a number of input data (e.g. some mechanical or chemical property). Although there are results in the field of engineering problems based on SVR models [12], this method is not yet particularly widespread for engineering optimization. The application of SVR in non-linear models has the advantage that the transformation function between input space and the so-called feature space (where a linear regression problem is to be solved) can be hidden [13], and machine learning procedures can be applied to find an appropriate regression function.

The novel procedure based on the FEM and SVR is suitable for the shape optimization of rubber bumpers with the specified characteristics. The efficiency of the method is verified by examples.

\section{THE OPTIMIZATION METHOD}

\subsection{The Objective Function of the Shape Optimization}

In the optimization process, we start from the load-displacement curve of an existing and known construction (Fig. 3). The shape optimization can be formulated as a minimization problem on a given domain by the following. The objective function $\Delta W: \Omega \rightarrow R$ gives the area between the desired loaddisplacement curve and the curve obtained by finite element computation for a specific rubber bumper shape (represented by the parameter vector $\mathbf{d}$ ):

$$
\Delta W(\mathbf{d})=\int_{0}^{s_{0}}\left|F_{\mathrm{des}}(s)-F_{\mathrm{FEM}, \mathbf{d}}(s)\right| d s,
$$

(the grey-filled area in Fig. 4). Function $\Delta W$ is considered on $\Omega \subset R^{n}$, the set of possible design parameter vectors $\mathbf{d}, s_{0}$ is the limit of the operation range, $F_{\mathrm{des}}$ and $F_{\mathrm{FEM}, \mathrm{d}}$ denote the spring force of the desired characteristics and the spring force calculated by finite element method for a specific geometry (determined by $\mathbf{d}$ ), respectively. The optimization range $\Omega$ is given by inequality conditions coming from technology limitations, $n$ is the number of the design parameters. Therefore, many design parameters are chosen, so many variables will be in the optimization.

Function $\Delta W$ has to be minimized by determining the optimum design parameter vector dopt, that is: 


$$
\Delta W\left(\mathbf{d}^{\mathrm{opt}}\right)=\min _{\mathbf{d} \in \Omega} \Delta W(\mathbf{d})
$$

Since numerical methods are used, the process results in an approximate value of the optimum.

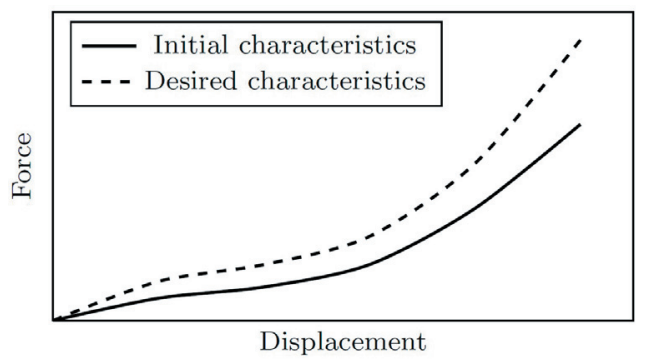

Fig. 3. The optimization task

The first step of the method is to solve a non-linear regression problem for function $\Delta W$. The regression procedure is based on the points $\left(\mathbf{d}^{i}, \Delta W\left(\mathbf{d}^{i}\right)\right) \in R^{n+1}, i=1, \ldots, N \quad$ (learning points), where $\Delta W\left(\mathbf{d}_{i}\right)$ are given (measured or calculated with FEM). The SVR model is used to find the regression function; the calculations are carried out with the SVR package of "R" software [14]. Since the values of the regression function provided by the software are available for arbitrary design parameter vectors in $\Omega$, the place of the minimum of $\Delta W$, i.e. the value of the optimum design parameter vector, can be determined numerically.

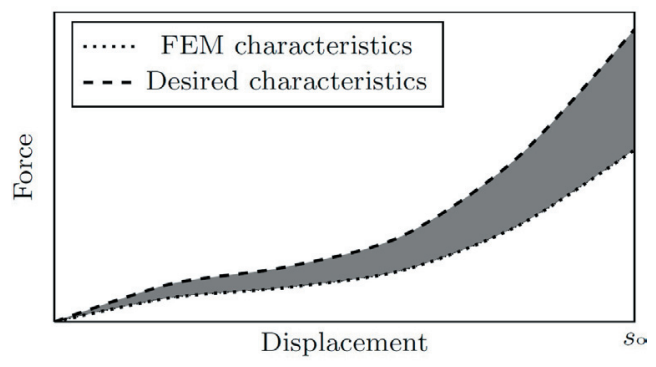

Fig. 4. Derivation of the objective function

\subsection{Application of the SVR Model}

The support vector regression model related to the theory of learning machines and kernel methods and widely used in statistics and lately in engineering calculations also plays a central role in our investigations. In this part, the theoretical background of the method is summarized briefly.

Using the finite element method, the values of $\Delta W$ are determined for the design parameters $\mathbf{d}^{1}, \ldots, \mathbf{d}^{N} \in \Omega, \quad$ and the learning points $\left(\mathbf{d}^{i}, \Delta W\left(\mathbf{d}^{i}\right)\right) \in R^{n+1}, i=1, \ldots, N$ play the role of the given data points in the (non-linear) regression procedure.

In the SVR model, the so-called kernel functions play a central role. Let $\mathbf{t}: \Omega \rightarrow \Omega^{\prime} \subset R^{m}$ be a transformation function mapping the learning points from the input space into what is called the feature space that transforms our non-linear problem into a linear one. It is widely used since the regression function can be expressed as a linear combination of kernel functions

$$
k_{i}(\mathbf{d})=\mathbf{t}(\mathbf{d})^{T} \cdot \mathbf{t}\left(\mathbf{d}^{i}\right),
$$

where ( $)^{T}$ denotes the transpose (see in [11]).

The regression function $f$ is looked for in the form:

$$
f(\mathbf{t}(\mathbf{d}))=\mathbf{w}^{T} \cdot \mathbf{t}(\mathbf{d})+b
$$

in the feature space, where $b \in R$ and $\mathbf{w} \in R^{m}$ . In the classic models, the optimization is based on the difference between the values of the regression function and the given values at the learning points $L\left(\mathbf{d}^{i}\right)=\left|f\left(\mathbf{t}\left(\mathbf{d}^{i}\right)\right)-\Delta W\left(\mathbf{d}^{i}\right)\right|$, which does not provide a satisfactory solution in many cases. In some cases, it appears to be better to use the $\varepsilon$-insensitive (Vapnik's) error function $L_{\varepsilon}$ to solve the regression problem [11] defined by:

$$
L_{\varepsilon}\left(\mathbf{d}^{i}\right)=\left\{\begin{array}{c}
0, \text { if }\left|f\left(\mathbf{t}\left(\mathbf{d}^{i}\right)\right)-\Delta W\left(\mathbf{d}^{i}\right)\right| \leq \varepsilon \\
\left|f\left(\mathbf{t}\left(\mathbf{d}^{i}\right)\right)-\Delta W\left(\mathbf{d}^{i}\right)\right|-\varepsilon, \text { otherwise }
\end{array},\right.
$$

where $\varepsilon$ is a fixed non-negative parameter. According to Eq. (5), the error is zero if the value of a learning point is in the $\varepsilon$-insensitive tube around the regression function (Fig. 5). $\varepsilon$ may be regarded as the parameter controlling the smoothness of the solution.

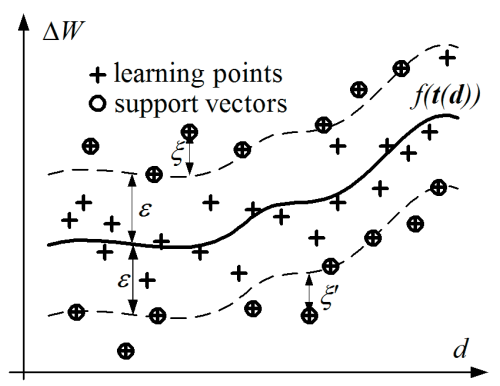

Fig. 5. $\varepsilon$-insensitive tube in $1 \mathrm{D}$ case

Using the $\varepsilon$-insensitive error function, the optimization problem leads to a constraint quadratic optimization problem, minimize: 


$$
\frac{1}{2} \mathbf{w}^{T} \cdot \mathbf{w}
$$

subject to:

$$
\left|\Delta W\left(\mathbf{d}^{i}\right)-\left(\mathbf{w}^{T} \cdot \mathbf{t}\left(\mathbf{d}^{i}\right)+b\right)\right| \leq \varepsilon, \quad i=1, \ldots, N .
$$

When using regression models, we have to take into consideration that certain learning points will disturb the run of the regression function; therefore, it is expedient to moderate the effect of such high values by means of the slack variables $\xi_{i}$ and $\xi_{i}$ : the difference of the ith learning point from the function should not be more than $\varepsilon+\xi_{i}$ and $\varepsilon+\xi_{i}{ }_{i}$, respectively. The higher the value of the slack variables, the larger the scope for searching for the function. Introducing the slack variables, the following modified constraint quadratic optimization problem is to be solved (see, e.g. [11]), minimize:

$$
\frac{1}{2} \mathbf{w}^{T} \cdot \mathbf{w}+C \sum_{i=1}^{N}\left(\xi_{i}+\xi_{i}^{\prime}\right)
$$

subject to:

$$
\begin{gathered}
\Delta W\left(\mathbf{d}^{i}\right)-\left(\mathbf{w}^{T} \cdot \mathbf{t}\left(\mathbf{d}^{i}\right)+b\right) \leq \varepsilon+\xi_{i}, \\
\left(\mathbf{w}^{T} \cdot \mathbf{t}\left(\mathbf{d}^{i}\right)+b\right)-\Delta W\left(\mathbf{d}^{i}\right) \leq \varepsilon+\xi_{i}{ }^{\prime} \\
\xi_{i} \geq 0, \xi_{i}{ }^{\prime} \geq 0, \quad i=1, \ldots, N .
\end{gathered}
$$

$C$ determines the trade-off between the error and the complexity of the solution. The hyper-parameter $C$ can be regarded as a penalty parameter that penalizes excessive divergence (larger than $\varepsilon$ ). For higher values of $C$, the objective function is more sensitive to the slack variables, so in the optimum solution the value of the slack variables remains low, and the function will be a reasonable approximation of the learning points, while a low value of $C$ can result in a function that runs at a considerable distance from certain learning points.

According to the standard dualization method [15], we introduce the Lagrange function:

$$
\begin{aligned}
& L\left(\mathbf{w}, b, \lambda, \lambda^{\prime}, \alpha, \alpha^{\prime}\right)= \\
& =\frac{1}{2} \mathbf{w}^{T} \cdot \mathbf{w}+C \sum_{i=1}^{N}\left(\xi_{i}+\xi_{i}^{\prime}\right)-\sum_{i=1}^{N}\left(\lambda_{i} \xi_{i}+\lambda_{i}^{\prime} \xi_{i}^{\prime}\right)- \\
& -\sum_{i=1}^{N} \alpha_{i}\left(\varepsilon+\xi_{i}+\left(\mathbf{w}^{T} \cdot \mathbf{t}\left(\mathbf{d}^{i}\right)+b\right)-\Delta W\left(\mathbf{d}^{i}\right)\right)- \\
& -\sum_{i=1}^{N} \alpha_{i}^{\prime}\left(\varepsilon+\xi_{i}^{\prime}+\Delta W\left(\mathbf{d}^{i}\right)-\left(\mathbf{w}^{T} \cdot \mathbf{t}\left(\mathbf{d}^{i}\right)+b\right)\right),
\end{aligned}
$$

where $\alpha_{i}, \alpha_{i}{ }^{\prime}, \lambda_{i}, \lambda_{i}{ }^{\prime} \geq 0$. The dual optimization problem is the following, maximize:

$$
\begin{aligned}
& -\frac{1}{2} \sum_{i, j=1}^{N}\left(\alpha_{i}-\alpha_{i}{ }^{\prime}\right)\left(\alpha_{j}-\alpha_{j}{ }^{\prime}\right) \mathbf{t}\left(\mathbf{d}^{i}\right)^{T} \cdot \mathbf{t}\left(\mathbf{d}^{j}\right) \\
& -\varepsilon \sum_{i=1}^{N}\left(\alpha_{i}+\alpha_{i}{ }^{\prime}\right)+\sum_{i=1}^{N}\left(\alpha_{i}-\alpha_{i}{ }^{\prime}\right) \Delta W\left(\mathbf{d}^{i}\right)
\end{aligned}
$$

subject to:

$$
\sum_{i=1}^{N}\left(\alpha_{i}-\alpha_{i}{ }^{\prime}\right)=0 \quad \text { and } \quad \alpha_{i}, \alpha_{i}^{\prime} \in[0, C] .
$$

Solving the dual problem, we obtain:

$$
f(\mathbf{t}(\mathbf{d}))=\sum_{i=1}^{N}\left(\alpha_{i}-\alpha_{i}{ }^{\prime}\right) \mathbf{t}(\mathbf{d})^{T} \cdot \mathbf{t}\left(\mathbf{d}^{i}\right)+b,
$$

that is, the solution (regression function) is a linear combination of kernel functions $k_{i}(\mathbf{d})=\mathbf{t}(\mathbf{d})^{T} \cdot \mathbf{t}\left(\mathbf{d}^{i}\right)$.

This form of $f$ says that the explicit form of $\mathbf{w}$ does not need to be computed. Furthermore, it can be proved that for the learning points inside the $\varepsilon$-tube $\alpha_{i}-\alpha_{i}{ }^{\prime}=0$, that is $f$ is determined only by the learning points having non-vanishing coefficients. These pairs are called support vectors.

Which learning points play the parts of support vectors depends on the choice of parameter $\varepsilon$ (Fig. 5). The wider the band, the smoother the solution. Otherwise, the model will attempt to fit the solution more accurately to the learning points, and the function will change more rapidly. The fact that the solution is determined by support vectors is a consequence of using the special error function (Eq. (5)).

It is known that the solution of the problem discussed above is a regression function, which can be written as a linear combination of kernel functions $k_{i}, i=1, \ldots, N$ having the form of Eq. (3), so we are not involved with the transformation function $\mathbf{t}$. This fact leads to the following idea: choosing a suitable kernel function, an appropriate solution of the regression problem can be achieved.

In accordance with our calculation experience and the research results in this field, the Gaussian kernel function:

$$
k_{i}(\mathbf{d})=e^{-\gamma \mid \mathbf{d}-\mathbf{d}^{i} \|},
$$

belonging to the class of radial basis functions (RBF) provides the best solution to our problem, where $\gamma$ is a parameter controlling the form of the kernel function. In the calculation, the Gaussian function Eq. (16) is chosen as a kernel function. Since it depends only on 
the distances $\left\|\mathbf{d}-\mathbf{d}_{i}\right\|, i=1, . ., N$, the learning points behave as a kind of centre when the Gaussian kernel functions are applied. The value of the regression function is fundamentally determined by the nearby known values (learning points).

In the following, the regression function obtained from the SVR model is denoted by $\Delta W_{\mathrm{SVR}}$, that is:

$$
\Delta W_{\mathrm{SVR}}(\mathbf{d})=f(\mathbf{t}(\mathbf{d}))
$$

It is easy to see that the accuracy of the regression provided by the SVR method depends on $\gamma$ and $C$. An optimum choice of the hyper-parameters $(\gamma, C)$ leads to the error of the learning process:

$$
\delta_{1}=\sqrt{\frac{\sum_{i=1}^{N}\left(\Delta W_{\mathrm{FEM}}^{i}-\Delta W_{\mathrm{SVR}}^{i}\right)^{2}}{\sum_{i=1}^{N}\left(\Delta W_{\mathrm{FEM}}^{i}\right)^{2}}} \leq \delta_{1}^{*},
$$

where $\Delta W_{\mathrm{SVR}}^{i}=\Delta W_{\mathrm{SVR}}\left(\mathbf{d}^{i}\right)$ and $\Delta W_{\mathrm{FEM}}^{i}=\Delta W_{\mathrm{FEM}}\left(\mathbf{d}^{i}\right)$ are the values of the objective function determined by the finite element calculation. $\delta_{1}{ }^{*}$ can be specified by the user according to the expected accuracy. A successful combination of hyper-parameters can be achieved when the error remains within the specified $\operatorname{limit} \delta_{1}{ }^{*}$.

In our examples, the hyper-parameters are chosen by the following steps:

- the initial values of the hyper-parameters $\gamma_{\min }$, $C_{\min }$ are predefined;

- while keeping $C=C_{\min }$ at a constant value, we are considering the error $\delta_{1}$ as a function of $\gamma$ and looking for the parameter $\gamma=\gamma_{\text {opt }}$, where $\delta_{1}$ is minimal;

- hen keeping the parameter $\gamma_{\text {opt }}$ at a constant value, we are considering the error $\delta_{1}$ as a function of $C$ and looking for the parameter $C=C_{\mathrm{opt}}$, where $\delta_{1}$ is within the specified limit $\delta_{1}{ }^{*}$;

- values of $\gamma_{\mathrm{opt}}$ and $C_{\mathrm{opt}}$ mean the hyper-parameters satisfy the requirements in Eq. (18) and determine the regression function used in the last part of the shape optimization process.

Considering a set:

$$
D=\left\{\mathbf{d}^{1}, \ldots, \mathbf{d}^{P}\right\} \subset \Omega,
$$

according to technology limitations the minimum of $\Delta W_{\mathrm{SVR}}$ on $D$ is determined numerically.

\section{PRODUCING THE LEARNING POINTS BY THE FEM}

Rubber bumpers may undergo large deformations under load, which in itself shows non-linear behaviour. The changing contact range between the parts and the incompressibility of the rubber increases this nonlinear behaviour further.

In order to be able to use the SVR method, the spring characteristics have to be produced by the finite element code within the optimization range, and then the difference of the work values has to be calculated for the learning points to be written. There is no generally accepted rule concerning the learning points. Their number may depend on the expected accuracy and the type of the problem, among other factors. In determining the learning points, the objective was that they should properly cover the optimization range.

For rubber, the material models are generally given by the strain energy density function [16]. The energy density function of nearly incompressible materials can be divided into a volume-changing and a volume-preserving part. The strain energy density resulting from the change in volume $U(J)$ is given in the following form:

$$
U(J)=\frac{1}{2} \cdot \kappa \cdot(J-1)^{2},
$$

where $J$ is the Jacobian, $\kappa$ is the bulk modulus, which is a real material characteristic and in the finite element investigations can be interpreted as a penalty parameter. If an incompressible material is examined, then $U(J)$ is zero, for $v=0.5$. It is to be noted that rubber bumpers may be regarded as nearly incompressible materials due to additives. Accordingly, the Poisson ratio is between $0.49<v<0.5$.

A compression test according to the standard ISO 7743 is performed to determine the stressstrain curve of the rubber part. A number of material models can be found in the literature for the volumepreserving member of strain energy density. Since the deformation is relatively small in our investigations, the two-parameter Mooney-Rivlin material model is sufficient to describe the material behaviour, where the strain energy density is expressed using the scalar invariants:

$$
\widehat{W}(\widehat{\mathbf{C}})=\mu_{10} \cdot\left(\hat{I}_{\mathrm{I}}-3\right)+\mu_{01} \cdot\left(\hat{I}_{\mathrm{II}}-3\right) .
$$

The Mooney-Rivlin material constants $\mu_{10}$ and $\mu_{0}$ are determined from the results of the compression test using FEM computations [7]. $\hat{I}_{\mathrm{I}}$ and $\hat{I}_{\text {II }}$ are the scalar invariants of the volume preserving member of the Cauchy-Green strain tensor of the right $C[16]$. 
The program developed uses the combined technique, which is based on the following functional:

$$
\begin{aligned}
& \Pi(\mathbf{u}, \bar{J}, \bar{p})=\int_{V} \widehat{W}(\widehat{\mathbf{C}}) d V+\int_{V} U(\bar{J}) d V+ \\
& +\int_{V} p \cdot(J-\bar{J}) d V+\frac{1}{2} \int_{A_{c}} c \cdot g_{n}^{2} d A-\Pi_{\text {ext }}(\mathbf{u}),
\end{aligned}
$$

where the displacement field $\mathbf{u}$ is approximated using the quadratic tensor product space, the volume change $\bar{J}$ and the hydrostatic pressure $\bar{p}$ are approximated using linear functions independently of each other, $U(\bar{J})$ is the penalty parameter member, $J$ can be indirectly derived from the displacement and is independent of $\bar{J}, c$ is the penalty parameter of the contact, $g_{n}$ is the gap function, $\Pi_{\text {ext }}(\mathbf{u})$ is the potential of the external forces, $V$ is the volume of the rubber and $A_{c}$ is the contact surface.

To discretize the functional, nine-node isoparametric axi-symmetric finite elements are used. Applying the Total-Lagrange description to the variation equations of functional Eq. (22) according to $\mathbf{u}, \bar{J}, \bar{p}$ [16], after finite element discretization, and the Newton-Raphson iteration equation [17] is finally obtained:

$$
\mathbf{K}_{T} \Delta \mathbf{u}=\Delta \mathbf{f}
$$

where $\mathbf{K}_{T}$ is the structural tangent stiffness matrix, $\Delta \mathbf{u}$ is the vector of nodal point displacement increment and $\Delta \mathbf{f}$ is the unbalanced load vector. The validation and calibration of our program (mesh, material constants and finite element input data) were performed according to [18]. For the finite element code, a data generation program (mesh, boundary conditions, loads and finite element input data) has also been developed.

\section{OPTIMIZATION PROCESS}

In the course of the optimization process, the geometry of the initial design, the results of the compression testing, and the specified characteristics are known.

As the first step, the finite element model is built, and then the Mooney-Rivlin material constants $\left(\mu_{10}, \mu_{01}\right)$ are determined with consideration of the measurement results.

In order to decrease the running time, the clearest mesh is found at which the required calculation accuracy can be preserved. For the same specified material characteristics $\left(\mu_{10}, \mu_{01}, \kappa\right.$ and $\left.c\right)$, the learning points are created.

The learning points are used to test the error $\delta_{1}$ of the regression function produced by the software optimizing the hyper-parameters $\gamma$ and $C$. If the error $\delta_{1}$ is within the specified limit $\delta_{1}{ }^{*}$ the regression function is accepted and is used for further calculation. The minimum of $\Delta W_{\mathrm{SVR}}$ on $D$ defined in Eq. (19) is calculated.

The finite element calculation is performed again with the optimum design parameter vector determined using SVR method and the condition:

$$
\delta_{2}=\frac{\left|\Delta \mathrm{W}_{\mathrm{FEM}}\left(\mathbf{d}^{\mathrm{opt}}\right)-\Delta \mathrm{W}_{\mathrm{SVR}}\left(\mathbf{d}^{\mathrm{opt}}\right)\right|}{\Delta \mathrm{W}_{\mathrm{FEM}}\left(\mathbf{d}^{\mathrm{opt}}\right)} \leq \delta_{2}^{*},
$$

is checked, where the limit is specified by the user.

If Eq. (24) is fulfilled, the optimization is considered to be completed. Fig. 6 shows the flowchart of the shape optimization problem.

\section{NUMERICAL EXAMPLES}

\subsection{Two-Dimensional Shape Optimization}

The rubber part investigated is the bumper of an air spring used in buses. The meridian section of the air spring containing the rubber bumper is shown in Fig. 1. The air springs are designed so that the buses can "kneel" at bus stops, and the air spring goes flat. The rubber bumper rests against the bumper plate at that time.

In current practice, when a softer or a harder rubber bumper is needed, this is achieved by changing the rubber composition. Our task is to enable the described rubber bumper to produce a $15 \%$ harder characteristic under operating conditions. This is to be achieved by changing the shape of the rubber bumper.

Table 1. FEM input data

\begin{tabular}{lc}
\hline Mooney-Rivlin constant $\left(\mu_{10}\right)$ & $0.63 \mathrm{~N} / \mathrm{mm}^{2}$ \\
\hline Mooney-Rivlin constant $\left(\mu_{01}\right)$ & $0.1575 \mathrm{~N} / \mathrm{mm}^{2}$ \\
\hline Bulk modulus $(\kappa)$ & $1000 \mathrm{~N} / \mathrm{mm}^{2}$ \\
\hline Penalty parameter of contact $(c)$ & $1000 \mathrm{~N} / \mathrm{mm}^{2}$ \\
\hline Prescribed disp. inc. $(\Delta u)$ & $1 \mathrm{~mm}$ \\
\hline Number of load steps $(m)$ & 13 \\
\hline
\end{tabular}

In Fig. 1, it can be seen that the rubber bumper comes into contact with the top plate and the pin even under a small compression. The displacements are known on the lower and upper surfaces. The finite element code was developed so that it would be suitable to also consider normal contact. The shape optimization problem is two-dimensional in this case. The finite element input data are given in Table 1 , the initial geometry of the rubber bumper and the 
deformed shape of the original geometry are shown in Fig. 7.

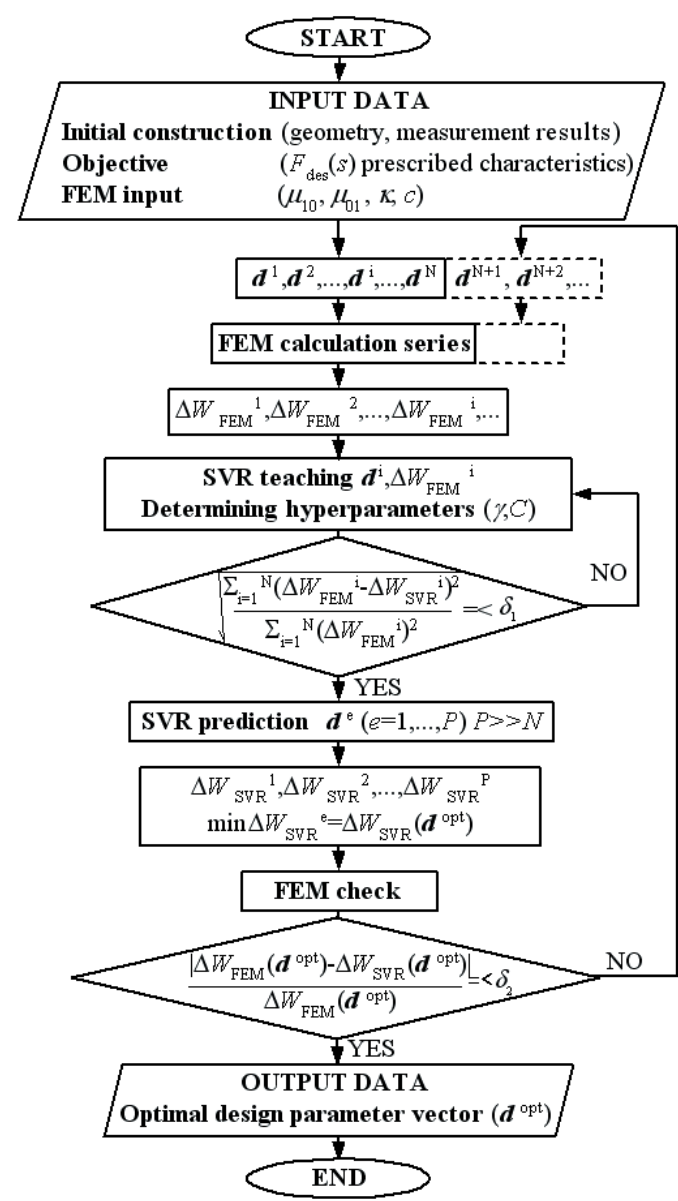

Fig. 6. Flowchart of the shape optimization

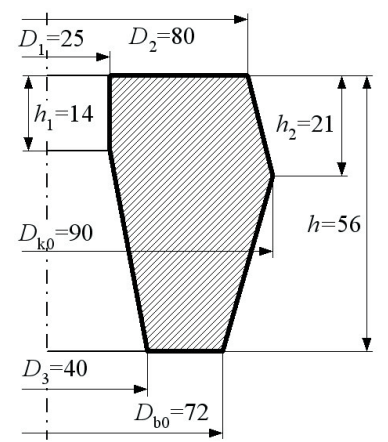

Fig. 7. The initial and the deformed geometry

The initial and the specified characteristics are shown in Fig. 8. Taking the production technology and application technology limitations into consideration, the two design parameters are the largest diameter $d_{1}=D_{k}$ and the external diameter of the bumper surface $d_{2}=D_{b}$. It is a fundamental requirement that the hole diameter $D_{1}$ and the height of the part $h$ should not change.

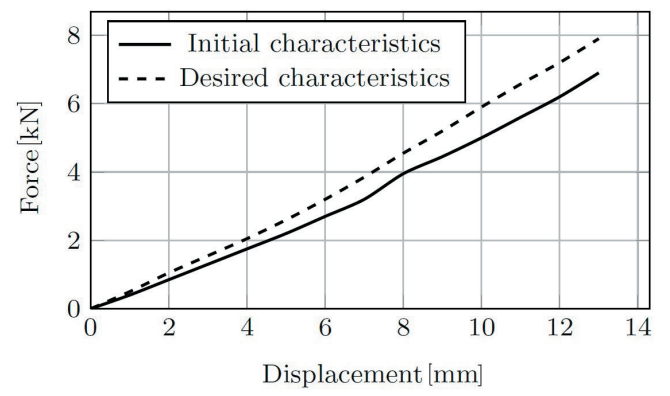

Fig. 8. The spring characteristics

In the shape optimization, the inequalities $d_{2} \geq D_{b 0}$ and $d_{1}>d_{2}$ are specified, so that the design parameters in $\mathrm{mm}$ are defined according to the following conditions:

$\mathbf{d}=\left(d_{1}, d_{2}\right)$, where $\left\{\begin{array}{l}d_{1} \in\{82,83, \ldots, 102\} \\ d_{2} \in\{74,75, \ldots, 90\}\end{array}\right.$ and $d_{1}>d_{2}$.

Thus, the optimization range and the learning points chosen can be seen in Fig. 9.

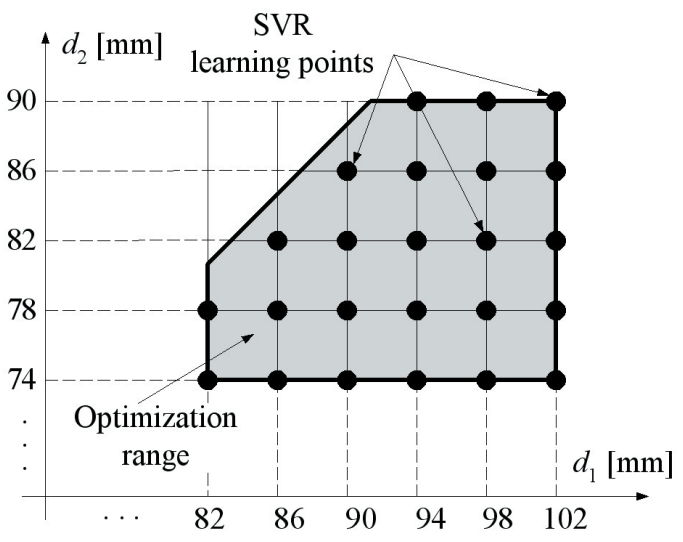

Fig. 9. The optimization region and the learning points

Table 2. Input data of the optimization process

\begin{tabular}{lc}
\hline SVR parameter $(\varepsilon)$ & 0.01 \\
\hline SVR parameter $\left(\gamma_{\min }\right)$ & 0.1 \\
\hline SVR parameter $\left(C_{\min }\right)$ & 1 \\
\hline Tolerance $\left(\delta_{1}{ }^{*}\right)$ & 0.01 \\
\hline Tolerance $\left(\delta_{2}{ }^{*}\right)$ & 0.05 \\
\hline
\end{tabular}

The manufacturing accuracy for the two design parameters is $1 \mathrm{~mm}$. Under the specified accuracy, the number of possible solutions is $P=312$, which can be determined from the predefined conditions on design 
parameters and manufacturing accuracy. The number of learning points is $N=24$. The input data of the optimization process are collected in Table 2.

After reading in the learning points on the basis of the hyper-parameter search (Figs. 10 and 11), the following parameters are considered to be optimal in the SVR program: $\gamma_{\mathrm{opt}}=1.5, C_{\mathrm{opt}}=1.5$, where $\delta_{1}=0.008429$. The goodness of learning is shown in Fig. 12.

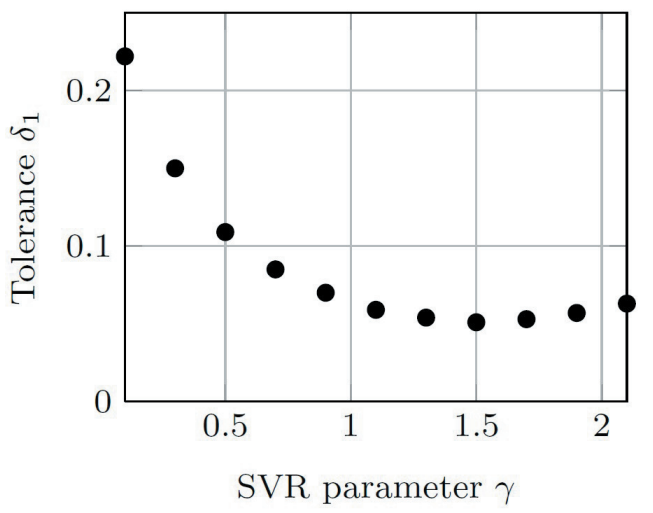

Fig. 10. Determination of $\gamma_{\text {opt }}\left(C_{\min }=\right.$ const. $)$

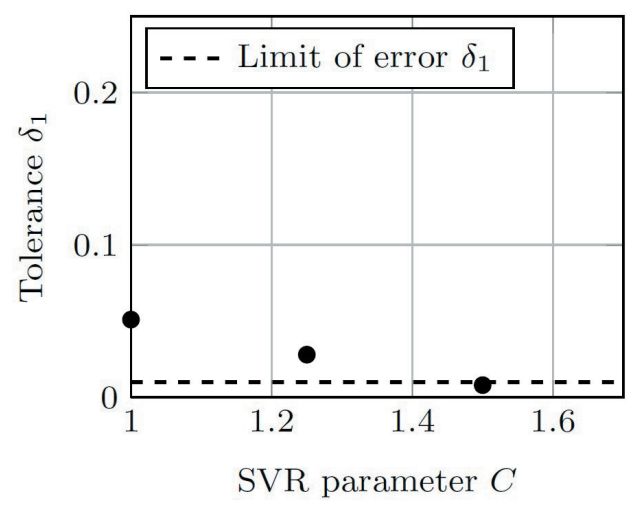

Fig. 11. Determination of $C_{\text {opt }}\left(\gamma_{\text {opt }}=\right.$ const. $)$

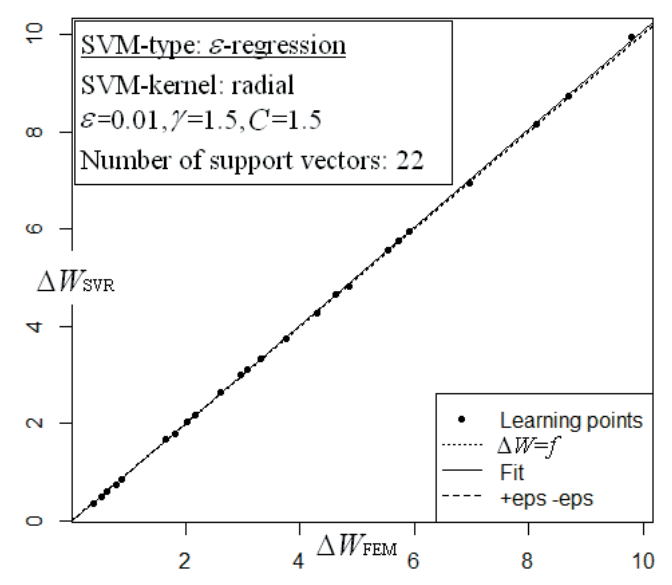

Fig. 12. SVR best fit
The results obtained by the teaching using the optimal hyper-parameters are collected in Table 3.

Table 3. Results of the SVR teaching

\begin{tabular}{ccccc}
\hline No. & $d_{1}[\mathrm{~mm}]$ & $d_{2}[\mathrm{~mm}]$ & $\Delta W_{\text {FEM }}[\mathrm{Nm}]$ & $\Delta W_{\text {SVR }}[\mathrm{Nm}]$ \\
\hline 1 & 82 & 74 & 9.94111 & 9.799091 \\
\hline 2 & 82 & 78 & 8.72711 & 8.669142 \\
\hline 3 & 86 & 74 & 8.16311 & 8.135446 \\
\hline 4 & 86 & 78 & 6.93211 & 6.959917 \\
\hline$\ldots$ & $\ldots$ & $\ldots$ & $\ldots$ & $\ldots$ \\
\hline 23 & 102 & 86 & 4.26789 & 4.284846 \\
\hline 24 & 102 & 90 & 5.55889 & 5.531014 \\
\hline
\end{tabular}

On the basis of the calculation, the minimum work difference of the possible solutions is $\Delta W_{\text {SVR }}\left(\mathbf{d}^{\mathrm{opt}}\right)=0.368536 \mathrm{Nm}$, for which the optimum design parameters are $d_{1}$ opt $=94 \mathrm{~mm}$ and $d_{2}$ opt $=88$ $\mathrm{mm}$. The optimization results are summarized in Table 4.

Table 4. Design solutions obtained with the SVR

\begin{tabular}{cccc}
\hline No. & $d_{1}[\mathrm{~mm}]$ & $d_{2}[\mathrm{~mm}]$ & $\Delta W_{\text {SVR }}[\mathrm{Nm}]$ \\
\hline 174 & 94 & 88 & 0.368536 \\
\hline 296 & 102 & 74 & 0.388816 \\
\hline 175 & 94 & 89 & 0.389079 \\
\hline 297 & 102 & 75 & 0.411857 \\
\hline 280 & 101 & 75 & 0.415206 \\
\hline 235 & 98 & 81 & 0.44596 \\
\hline$\ldots$ & $\ldots$ & $\ldots$ & $\ldots$ \\
\hline
\end{tabular}

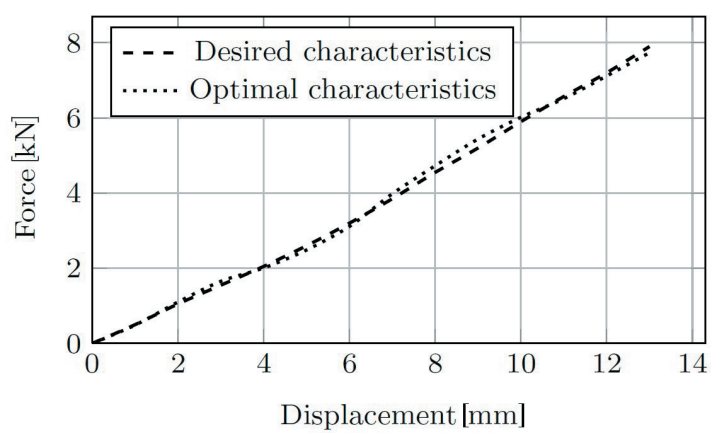

Fig. 13. The spring characteristics

The characteristics obtained for the control finite element calculation run for the optimum design variable and the specified characteristics are shown in Fig. 13, where $\Delta W_{\mathrm{FEM}}(\mathbf{d}$ opt $)=0.385546 \mathrm{Nm}$, so the tolerance is $\delta_{2}=0.04412$.

The optimal shape is shown in Fig. 14. 


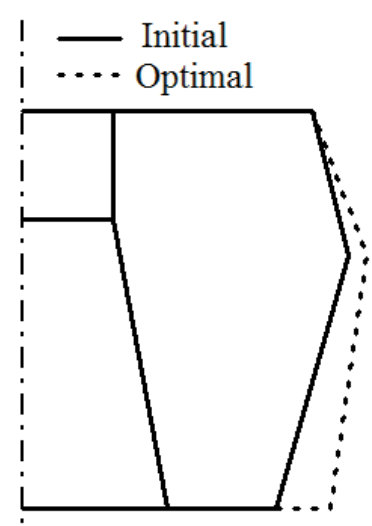

Fig. 14. The optimum shape

\subsection{Multi-Dimensional Shape Optimization}

Although the prescribed characteristics can be achieved by changing two or three design parameters in engineering practice, it may be considered that more design variables are needed to describe the specific feature. The shape optimization using SVR is suitable for handling even more design parameters. In the following example, the efficiency of this method is presented using a five-dimensional shape optimization problem. The outer skirt of the rubber bumper investigated is described by means of a cubic spline in five control points. These control points are the design parameters. The finite element input data are given in Table 5; the initial geometry of the rubber bumper and the deformed shape for the original geometry are shown in Fig. 15. The initial and the desired loaddisplacement curves can be seen in Fig. 16.

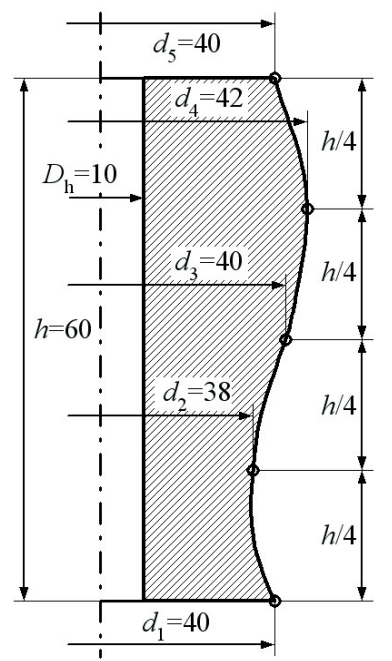

Fig. 15. The initial and the deformed geometry
Table 5. FEM input data

\begin{tabular}{lc}
\hline Mooney-Rivlin constant $\left(\mu_{10}\right)$ & $0.5 \mathrm{~N} / \mathrm{mm}^{2}$ \\
\hline Mooney-Rivlin constant $\left(\mu_{01}\right)$ & $0.125 \mathrm{~N} / \mathrm{mm}^{2}$ \\
\hline Bulk modulus $(\kappa)$ & $1000 \mathrm{~N} / \mathrm{mm}^{2}$ \\
\hline Prescribed disp. inc. $(\Delta u)$ & $2 \mathrm{~mm}$ \\
\hline Number of load steps $(m)$ & 10 \\
\hline
\end{tabular}

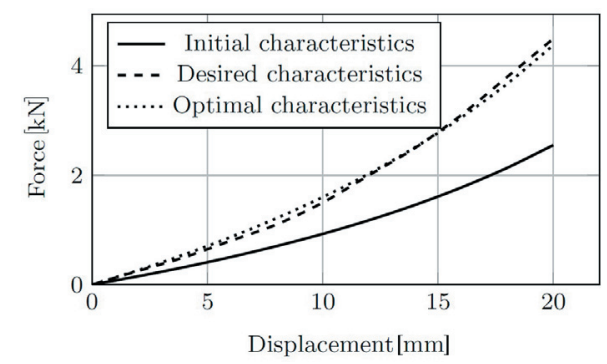

Fig. 16. The spring characteristics

In the investigation, the design parameters in $[\mathrm{mm}]$ are defined according to the following conditions:

$d=\left(d_{1}, d_{2}, d_{3}, d_{4}, d_{5}\right)$, where $\left\{\begin{array}{l}d_{1} \in[40,56] \\ d_{2} \in\left[d_{1}-4, d_{1}+4\right] \\ d_{3} \in\left[d_{2}-2, d_{2}+2\right] \\ d_{4} \in\left[d_{3}-2, d_{3}+2\right] \\ d_{5} \in\left[d_{4}-2, d_{4}+2\right]\end{array}\right.$

and $d_{1}, d_{2}, d_{3}, d_{4}, d_{5}$, are even numbers.

Under the specified accuracy, the number of possible solutions is $P=1215$. The number of learning points is $N=10$. The input data of the optimization process are included in Table 6.

Table 6. Input data of the optimization process

\begin{tabular}{lc}
\hline SVR parameter $(\varepsilon)$ & 0.01 \\
\hline SVR parameter $\left(\gamma_{\min }\right)$ & 0.05 \\
\hline SVR parameter $\left(C_{\min }\right)$ & 1 \\
\hline Tolerance $\left(\delta_{1}{ }^{*}\right)$ & 0.02 \\
\hline Tolerance $\left(\delta{ }^{*}{ }^{*}\right)$ & 0.05 \\
\hline
\end{tabular}

After reading in the learning points on the basis of the hyper-parameters search, the following parameters are considered to be optimum in the SVR program: $\gamma_{\mathrm{opt}}=0.1, C_{\mathrm{opt}}=20$, where $\delta_{1}=0.013775$. The goodness of learning is shown in Fig. 17.

On the basis of the calculation, the minimum work difference of the possible solutions is $\Delta W_{\mathrm{SVR}}\left(\mathbf{d o p t}^{\mathrm{ot}}\right)=0.432812 \mathrm{Nm}$, for which the optimum design parameters are $d_{1}$ opt $=52 \mathrm{~mm}, d_{2}$ opt $=48$ 
$\mathrm{mm}, d_{3}{ }^{\mathrm{opt}}=d_{4}{ }^{\mathrm{opt}}=d_{5} \mathrm{opt}=54 \mathrm{~mm}$. The results of the optimization are summarized in Table 7. The characteristics obtained for the control finite element calculation run for the optimum design variable and the specified characteristics are shown in Fig. 16, where $\Delta W_{\mathrm{FEM}}\left(\mathbf{d}_{\mathrm{pt} t}\right)=0.41267 \mathrm{Nm}$, so the tolerance is $\delta_{2}=0.04878$. A deformed shape for the optimal geometry is shown in Fig. 18.

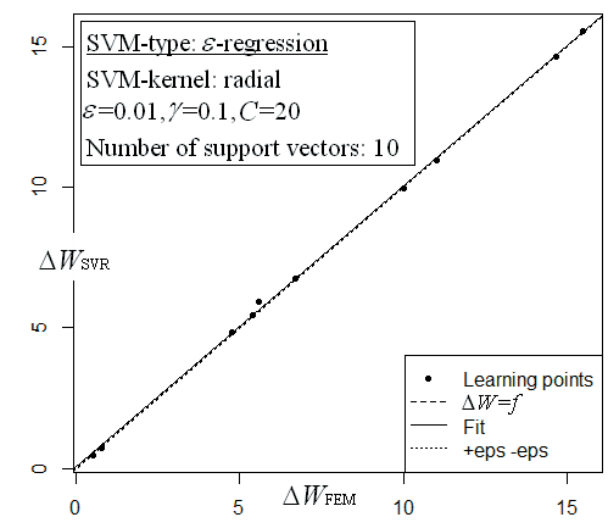

Fig. 17. SVR best fit

Table 7. Design solutions obtained with the SVR

\begin{tabular}{ccccccc}
\hline No. & $\begin{array}{c}d_{1} \\
{[\mathrm{~mm}]}\end{array}$ & $\begin{array}{c}d_{2} \\
{[\mathrm{~mm}]}\end{array}$ & $\begin{array}{c}d_{3} \\
{[\mathrm{~mm}]}\end{array}$ & $\begin{array}{c}d_{3} \\
{[\mathrm{~mm}]}\end{array}$ & $\begin{array}{c}d_{5} \\
{[\mathrm{~mm}]}\end{array}$ & $\begin{array}{c}\Delta W_{\text {SVR }} \\
{[\mathrm{Nm}]}\end{array}$ \\
\hline 837 & 52 & 48 & 54 & 54 & 54 & 0.43281 \\
\hline 861 & 52 & 50 & 54 & 52 & 54 & 0.43344 \\
\hline 828 & 52 & 48 & 52 & 54 & 54 & 0.45588 \\
\hline 836 & 52 & 48 & 54 & 54 & 52 & 0.4559 \\
\hline 852 & 52 & 50 & 52 & 52 & 54 & 0.45601 \\
\hline 860 & 52 & 50 & 54 & 52 & 52 & 0.46177 \\
\hline$\ldots$ & $\ldots$ & $\ldots$ & $\ldots$ & $\ldots$ & $\ldots$ & $\ldots$ \\
\hline & & & & & &
\end{tabular}

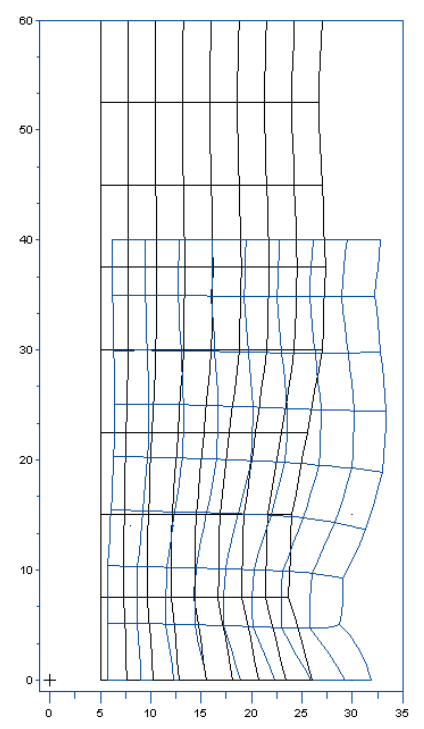

Fig. 18. Deformed shape of the optimal geometry

\section{CONCLUSIONS}

Rubber bumpers that are built into vehicles and structures have to meet several requirement as a result of their function. In this paper, bumpers with predefined load-displacement curves were achieved via shape optimization.

The characteristics of the rubber bumpers of different shapes were determined with the help of the finite element method. In the investigations, the SVR was used by means of open-source software to perform the optimization task. Combining the above two methods into one system, two shape optimization problems were solved to prove the efficiency of the presented procedure for axi-symmetric rubber bumpers.

The SVR method requires relatively few timeconsuming learning points to treat non-linear multidimensional optimization problems. Naturally, the learning points should cover the optimization range. The density of the points may depend on the complexity of the problem.

The teaching procedure producing a small number of learning points and carried out by using the finite element method can be regarded as short. After the teaching procedure, the software provides a remarkably strong prediction for further multitude of parameters by dispensing with the time-consuming finite element calculations and relying on engineering intuition.

For both two and five design parameters, the novel shape optimization procedure proved to be fast and accurate (see the examples presented in 4.1 and 4.2 , respectively). This regression process results in an approximation value of the objective function. The goodness of the calculation can be checked by finite element computation. Experimental measurements on bumper shapes obtained by numerical simulations would be justified, but this was not part of the current project.

It may be considered that further work is needed on multi-objective optimization including total mass or life cycle number, etc.

\section{REFERENCES}

[1] Gáspár, P., Szászi, I., Bokor, J. (2003). Design of robust controllers for active vehicle suspension using the mixed synthesis. Vehicle System Dynamics, vol. 40, no. 4, p. 193-228.

[2] Luo, R.K., Wu, W.X. (2006). Fatigue failure analysis of antivibration rubber spring. Engineering Failure Analysis, vol. 13, no. 1, p. 110-116. 
[3] Li, Q., Zhao, J., Zhao, B. (2009). Fatigue life prediction of a rubber mount based on test of material properties and finite element analysis. Engineering Failure Analysis, vol. 16, no. 7, p. 2304-2310.

[4] Oman, S., Fajdiga, M., Nagode, M. (2010). Estimation of air-spring life based on accelerated experiments. Materials and Design, vol. 31, no. 8, p. 3859-3868.

[5] Oman, S., Nagode, M. (2013). On the influence of the cord angle on air-spring fatigue life. Engineering Failure Analysis, vol. 27, p. 61-73.

[6] Wang, L.R., Wang, J.C., Hagiwara, I. (2005). An integrated characteristic simulation method for hydraulically damped rubber mount of vehicle engines. Journal of Sound and Vibration, vol. 286, no. 4-5, p. 673-696.

[7] Kim, J.J., Kim, H.Y. (1997). Shape design of an engine mount by a method of parameter optimization. Computers and Structures, vol. 65, no. 5, p. 725-731.

[8] Choi, K.K., Duan, W. (2000). Design sensitivity analysis and shape optimization of structural components with hyperelastic material. Computer Methods in Applied Mechanics and Engineering, vol. 187, p. 219-243.

[9] Ramachandran, T., Padmanaban, K.P., Nesamani, P. (2012). Modeling and analysis of IC engine mount using finite element method and RSM. Procedia Engineering, vol. 38, p. 1683-1692.
[10] Lee, J.S., Kim, S.C. (2007). Optimal design of engine mount rubber considering stiffness and fatigue strength. Journal of Automobile Engineering, vol. 221, no. 7, p. 823-835.

[11] Drucker, H., Bruges, C.J.C., Kaufman, L., Smola, A.J., Vapnik, V. (1997) Support vector regression machines. Advances in Neural Information Processing System, vol. 9, p. 155-161.

[12] Andrés, E., Salcedo-Sanz, S., Monge, F., Pérez-Bellido, A.M. (2012). Aerodynamic design through evolutionary programming and support vector regression algorithm. Expert Systems with Applications, vol. 39, no. 12, p. 10700-10708.

[13] Haykin, S. (2009). Neural networks and learning machines. Pearson Prentice Hall, Upper Saddle River.

[14] Karatzoglou, A., Meyer, D., Hornik, K. (2006). Support vector machines in R. Journal of Statistical Software, vol. 15 , no. 9 , p. 1-28.

[15] Boyd, S., Vandenberghe, L. (2009). Convex Optimization. Cambridge University Press, Cambridge.

[16] Bonet, J., Wood, R.D. (1997). Nonlinear continuum mechanics for finite element analysis. Cambridge University Press, Cambridge, p. 248.

[17] Bathe, K.J. (1996). Finite Element Procedures. Prentice Hall, New Jersey.

[18] Mankovits, T., Szabó, T. (2012). Finite element analysis of rubber bumper used in air-spring. Procedia Engineering, vol. 48, p. 388-395. 